\title{
A RELATIVE NASH THEOREM
}

\author{
BY
}

SELMAN AKBULUT AND HENRY C. KING

\begin{abstract}
We prove that if $M$ is a closed smooth manifold and $M_{i}, i=1, \ldots, k$, are transversally intersecting closed smooth submanifolds of $M$, then there exist a nonsingular algebraic set $Z$ and nonsingular algebraic subsets $Z_{i}, i=1, \ldots, k$, of $Z$ such that $\left(M ; M_{1}, \ldots, M_{k}\right)$ is diffeomorphic to $\left(Z ; Z_{1}, \ldots, Z_{k}\right)$. We discuss a generalization and the consequences of this result.
\end{abstract}

1. Introduction. In $1952 \mathrm{Nash}$ proved that every smooth closed manifold is a component of a nonsingular algebraic set ${ }^{1}[\mathrm{~N}]$; and he conjectured that it can in fact be made a nonsingular algebraic set. In 1973 Tognoli verified this conjecture [T]. We will prove a "relative" version of this result which in particular implies the following:

THEOREM 1.1. Let $M$ be a closed smooth manifold, and let $M_{i}, i=1, \ldots, k$ be closed smooth submanifolds of $M$ in general position. Then there is a nonsingular algebraic set $Z$ and nonsingular algebraic subsets $Z_{i}, i=1, \ldots, k$, of $Z$ and $a$ diffeomorphism $\phi: M \rightarrow Z$ with $\phi\left(M_{i}\right)=Z_{i}, i=1, \ldots, k$.

Our main motivation for proving this theorem was to prove a converse to the resolution theorem of $[\mathbf{H}]$. The resolution theorem says that: if a closed smooth manifold $\Sigma$ is a link of a real algebraic set $Z$ with an isolated singularity (i.e. $Z \approx \operatorname{cone}(\Sigma)$ ); then $\Sigma$ bounds a compact smooth manifold $W$ with the property that $W$ has transversally intersecting closed smooth submanifolds $M_{i}, i=$ $1, \ldots, k$, with $W / \cup M_{i} \approx \operatorname{cone}(\Sigma)$ (i.e. $\cup M_{i}$ is a spine of $W$ ).
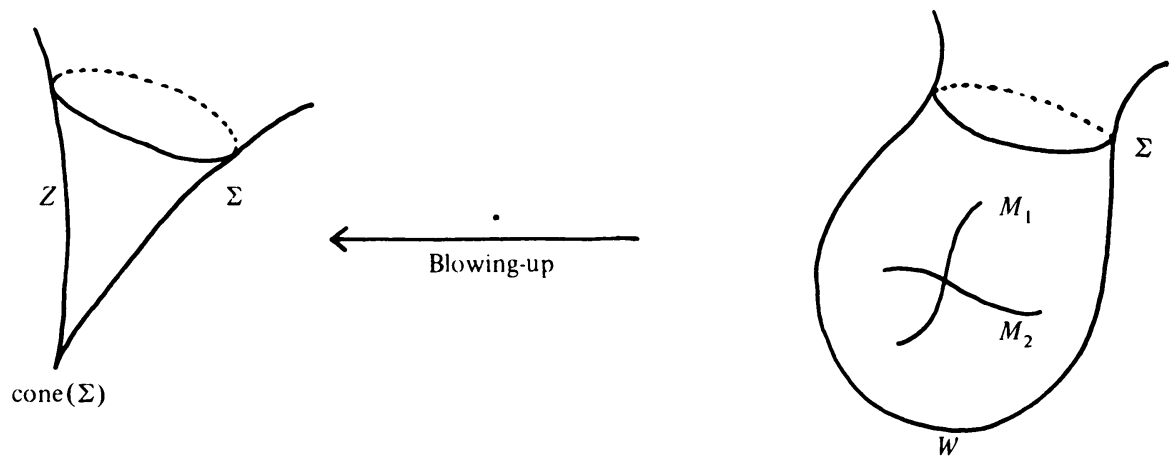

FigURE 1

Received by the editors December 12, 1979 and, in revised form, October 1, 1980. 1980 Mathematics Subject Classification. Primary 57C05, 14A10, 14E15, 57C25.

${ }^{1}$ Here algebraic set means a zero set of real polynomial functions. 
$W$ is obtained by successively blowing up the singular point of $Z$. Now by using Theorem 1.1 we can prove a converse to this. Namely, if we are given $\Sigma$ bounding such a $W$; we let $M=W \cup_{b}-W$; and then by the theorem we can make $\left(M, M_{1}, \ldots, M_{k}\right)$ diffeomorphic to nonsingular algebraic sets $\left(Z, Z_{1}, \ldots, Z_{k}\right)$. We can then algebraically collapse (blow down) the algebraic subsets $Z_{i}, i=1, \ldots, k$ of $Z$ to a point. Then we get an algebraic set $Z / \cup Z_{i} \approx M / \cup M_{i}$. In particular this makes cone $(\Sigma)$ an algebraic variety. This was outlined in $\left[\mathbf{A K} \mathbf{K}_{\mathbf{1}}\right]$. We proved Theorem 1.1 back in 1976 for this purpose. But since then, we have found a quick proof of this theorem under the additional assumption that each $M_{i}$ has codimension one $\left[\mathbf{A K}_{2}\right]$. We also proved that every bounding manifold $\Sigma$ must bound a $W$ which has a spine consisting of union of transversally intersecting closed codimension-one submanifolds $\boldsymbol{M}_{i}, i=1, \ldots, k\left[\mathbf{A K}_{\mathbf{2}}\right],\left[\mathbf{A K} \mathbf{K}_{3}\right]$. Hence in $\left[\mathbf{A K} \mathbf{K}_{2}\right]$ we were able to prove the result " $\Sigma$ is a link of an algebraic set with isolated singularity if and only if it bounds" without using Theorem 1.1. But Theorem 1.1 remains interesting because it gives many different ways of making cone $(\Sigma)$ an algebraic variety with an isolated singularity. This theorem can be useful for "classifying" all the algebraic sets, with an isolated singularity and a given link $\Sigma$.

There is an old question whether smooth closed submanifolds of a nonsingular algebraic set are isotopic to nonsingular algebraic subsets. Our theorem says that this can be done if we are allowed to move the ambient algebraic set by an isotopy (to another nonsingular algebraic set). As an application of Theorem 1.1 we prove that the interior of a smooth compact manifold with boundary is diffeomorphic to an open nonsingular algebraic set, which is properly imbedded in $\mathbf{R}^{n}$ for some $n$ (Corollary 2.11).

In $\$ 2$ we give some definitions and lemmas. We of ten refer to [AK $\mathbf{K}_{2}$ ] for proofs of some of these basic lemmas. We also state the main result (Theorem 2.10) in this section. In $\$ 3$, we give a quick proof of a weaker version of Theorem 1.1. We recommend that the reader should read this section before reading the main proof in $\S 4$. In $\S 2$ we make a conjecture which roughly says that up to diffeomorphism $\mathbf{Z} / 2 \mathbf{Z}$ homology classes of a nonsingular algebraic set are represented by algebraic subsets; if this conjecture turns out to be true then the proof in $\$ 3$ can be considered as the main proof. $\$ 3$ is a good warm-up exercise for $\$ 4$. Other than Corollary 2.11 the reader can mostly skip $\$ 2$ and use it as a reference section for definitions and lemmas used in the other sections.

\section{Preliminary lemmas.}

Definition. A real algebraic set is a set $V$ of the form $V(I)=\left\{x \in \mathbf{R}^{n} \mid p(x)=0\right.$, for all $p \in I\}$ where $I$ is a set of polynomial functions from $\mathbf{R}^{n}$ to $\mathbf{R}$.

In this paper "algebraic set" will always mean "real algebraic set".

DEFINITION. A point $x$ in an algebraic set $V \subset \mathbf{R}^{k}$ is called nonsingular of dimension $d$ in $V$ if there are polynomials $p_{i}(x), i=1, \ldots, k-d, x \in \mathbf{R}^{n}$ and a neighborhood $U$ of $x$ in $\mathbf{R}^{k}$ so that $p_{i}(V)=0$ and

(i) $V \cap U=U \cap \cap_{i=1}^{k-d} p_{i}^{-1}(0)$,

(ii) the gradients $\left(\nabla p_{i}\right)_{x}, i=1, \ldots, k-d$, are linearly independent on $U$.

We define $\operatorname{dim} V=\max \{d \mid$ there is an $x \in V$ which is nonsingular of dimension $d$ \}. 
DEFINITION. For an algebraic set $V$, Nonsing $V=\{x \in V \mid x$ is nonsingular of $\operatorname{dim} V\}$ and Sing $V=V-$ Nonsing $V$. We say that an algebraic set $V$ is nonsingular if Sing $V=\varnothing$.

Given a polynomial $p: \mathbf{R}^{n} \rightarrow \mathbf{R}$ of degree $d$ we associate a homogeneous polynomial $p^{*}: \mathbf{R} \times \mathbf{R}^{n} \rightarrow \mathbf{R}$ by $\dot{p}^{*}(t, x)=t^{d} p(x / t)=\sum_{i=0}^{d} t^{d-i} p_{i}(x)$ where $p_{i}$ is the sum of the monomials of $p$ of degree $i$. Let $\theta: \mathbf{R}^{n} \rightarrow \mathbf{R} P^{n}$ be the imbedding $\theta\left(x_{1}, \ldots, x_{n}\right)=\left(1: x_{1}: x_{2}: \cdots: x_{n}\right)$, and let $V\left(p^{*}\right) \subset \mathbf{R} P^{n}$ be the projective algebraic set given by the zeros of $p^{*}$. Then $V\left(p^{*}\right) \cap \theta\left(\mathbf{R}^{n}\right)=\theta\left(p^{-1}(0)\right)$. We say that the polynomial $p(x)$ is overt if $V\left(p^{*}\right)=\theta\left(p^{-1}(0)\right)$. This means that $p(x)$ does not "hide" zeros at infinity, or more precisely $p_{d}^{-1}(0)$ is $\{0\}$ or empty.

Definition. An algebraic set $V$ is called projectively closed if $V=p^{-1}(0)$ for some overt polynomial $p(x)$.

Hence an algebraic set $V$ is projectively closed if and only if $\theta(V)$ is a projective algebraic subset of $\mathbf{R} P^{n}$.

DEFINITION. Let $V$ and $W$ be algebraic sets and let $U$ be an algebraic subset of $V$. Let $W \subset \mathbf{R}^{n}$. Then a function $f: V-U \rightarrow W$ is called a rational function if there are polynomials $p: V \rightarrow \mathbf{R}^{n}$ and $q: V \rightarrow \mathbf{R}$ so that $q^{-1}(0) \subset U$ and $f(x)=p(x) / q(x)$ for all $x \in V-U$. If $U$ is empty then we call $f$ an entire rational function.

DEFINITION. Let $W$ be a smooth manifold. We say that a finite collection of closed submanifolds $M_{\alpha} \subset W, \alpha \in A$ are in general position if for each $\alpha \in A$ and $A^{\prime} \subset A-\alpha, M_{\alpha}$ intersects $\bigcap_{\alpha^{\prime} \in A^{\prime}} M_{\alpha^{\prime}}$ transversally.

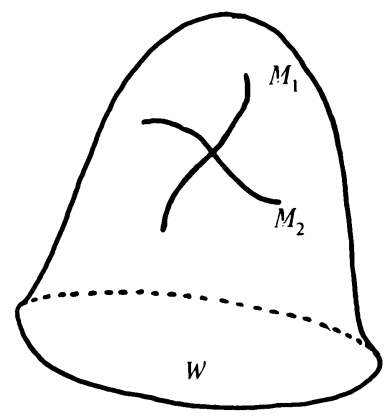

Figure 2

Next we state a few basic lemmas; they are useful for the main proofs. Lemmas 2.1, 2.2, 2.3, 2.4, 2.5, 2.6 correspond to Lemmas 2.9, 1.4, 1.6, 1.7, 2.1, 2.2 of [ $\left.\mathbf{A K}_{2}\right]$ respectively. Hence we refer the reader to $\left[\mathbf{A K}_{2}\right]$ for the proofs of these lemmas, except Lemma 2.4, which we prove here.

LEMMA 2.1. Let $M_{\alpha} \subset W, \alpha \in A$ be a finite collection of submanifolds in general position and $h_{t}^{\alpha}: M_{\alpha} \rightarrow W, t \in[0,1], \alpha \in A$ are small isotopies of imbeddings with $h_{0}^{\alpha}=$ inclusion. Then there is an isotopy $h_{t}: W \rightarrow W$ so that $h_{t}\left(M_{\alpha}\right)=h_{t}^{\alpha}\left(M_{\alpha}\right)$ for each $t \in[0,1]$ and $\alpha \in A$ and $h_{0}$ is the identity. $h_{t}$ can be arbitrarily small if the $h_{t}^{\alpha}$ are arbitrarily small.

REMARK. The isotopy $h_{t}$ covers $h_{t}^{\alpha}$ setwise, not pointwise. 
LEMMA 2.2. If $U, V, W$ and $X$ are algebraic sets with $X \subset V$ and $U \subset W$ and if $f: V-X \rightarrow W$ is a rational function, then $X \cup f^{-1}(U)$ is an algebraic set. Furthermore if $z \in f^{-1}(U)$ is nonsingular of dimension $r$ in $V$, and $f(z)$ is nonsingular of dimension $s$ in $U$ and nonsingular of dimension $t$ in $W$, and $f$ is transverse to $U$ at $z$, then $z$ is nonsingular of dimension $r+s-t$ in $f^{-1}(U)$.

LEMMA 2.3. Let $V \subset \mathbf{R}^{n}$ be an algebraic set and $W \subset$ Nonsing $V$ be an algebraic subset with $\operatorname{dim} W=\operatorname{dim} V$. Then $V-W \subset \mathbf{R}^{n}$ is an algebraic set. Also $\operatorname{Sing}(V-W)=\operatorname{Sing} V$ unless $W=$ Nonsing $V$.

LEMMA 2.4. Any compact algebraic set $V$ is the zero set of a proper polynomial (proper means that the inverse images of compacta are compact).

Proof. Let $V$ be a compact algebraic set. Let $p: \mathbf{R}^{n} \rightarrow \mathbf{R}$ a polynomial with $V=p^{-1}(0)$. Assume $p$ is not constant, otherwise the lemma is trivial. Let $B=\{x \in$ $\left.\mathbf{R}^{n}|| x \mid \leqslant R\right\}$; by taking $R$ large enough we can assume that $V \subset \operatorname{int}(B)$. Define $\theta$ : $\mathbf{R}^{n}-\{0\} \rightarrow \mathbf{R}^{n}-\{0\}$ by $\theta(x)=x /|x|^{2}$. Note that $\theta^{-1}=\theta$. Let $q(x)$ be the polynomial $q(x)=p^{*}\left(|x|^{2}, x\right)$. Then since $p^{-1}(0)$ is compact,

$$
q^{-1}(0)=\{0\} \cup \theta\left(p^{-1}(0)-\{0\}\right) .
$$

By the Lojasiewicz inequality [L] there are positive numbers $b$ and $a$ so that $|q(x)| \geqslant a|x|^{b}$ for all $x \in \theta\left(\mathbf{R}^{n}-B\right)$. Let degree $(p)=e$, then $q(x)=|x|^{2 e} p(\theta(x))$. Hence for all $x \in \mathbf{R}^{n}-B$

$$
\begin{aligned}
|p(x)| & =|\theta(x)|^{-2 e}|q(\theta(x))| \geqslant|\theta(x)|^{-2 e} a|\theta(x)|^{b} \\
& =a|\theta(x)|^{b-2 e}=a|x|^{2 e-b} .
\end{aligned}
$$

Hence $|p(x)| \geqslant a|x|^{d}$ for all $x \in \mathbf{R}^{n}-B$ where $d$ is some number, $a$ is a positive number. Then $p(x)\left(1+|x|^{2}\right)^{|d|+1}$ is a proper polynomial whose zero set is $V$.

LeMMA 2.5. Let $K \subset \mathbf{R}^{n}$ be a compact set and $L \subset K$ be an algebraic set. Let $f: \mathbf{R}^{n} \rightarrow \mathbf{R}$ be a smooth function so that for each $p$ in $L$ there is an algebraic set $M$ containing $L$ with $f(x)=0$ for all $x$ in $M$ near $p$, and $p$ is a nonsingular point of dimension $d$ in $M$ for some $d$. Then there is a polynomial $h:\left(\mathbf{R}^{n}, L\right) \rightarrow(\mathbf{R}, 0)$ which is arbitrarily close to $f$ near $K\left(C^{r}\right.$ close for a given $\left.r\right)$. In addition if $f$ has compact support to begin with, then $f$ can be approximated by an entire rational function $u:\left(\mathbf{R}^{n}, L\right) \rightarrow(\mathbf{R}, 0)$ on all of $\mathbf{R}^{n}$ (not just on a compact set $\left.K\right)$.

LEMMA 2.6. Let $W$ be an algebraic set and $M$ be a codimension 1 closed smooth submanifold of Nonsing $W$ which compactly separates $W$ (this means that $M$ separates $W$ into two components and one of these components is compact). Also let $L, V$ be algebraic sets with $L \subset$ Nonsing $V \subset M$. Then there is an arbitrarily small isotopy of $M$ in Nonsing $W$ which fixes $L$ and carries $M$ to a nonsingular algebraic set. If $L$ is projectively closed this isotopy carries $M$ to a nonsingular projectively closed set. 
Let $G(n, k)$ be the Grassmann manifold of $k$-planes in $\mathbf{R}^{n}$. Let $E(n, k) \rightarrow G(n, k)$ be the universal $\mathbf{R}^{k}$-bundle over $G(n, k)$. It is well known that $G(n, k)$ and $E(n, k)$ are nonsingular algebraic sets

$$
\begin{aligned}
& G(n, k)=\left\{A \in \mathbf{R}^{n^{2}} \mid A^{t}=A, A^{2}=A, \operatorname{trace} A=k\right\}, \\
& E(n, k)=\left\{(A, y) \in G(n, k) \times \mathbf{R}^{n} \mid A y=y\right\}
\end{aligned}
$$

where we view the elements of $\mathbf{R}^{n^{2}}$ as $(n \times n)$-matrices. Every such matrix $A \in$ $G(n, k)$ gives rise to a $k$-plane image $(A)$ and conversely to every $k$-plane we associate the projection matrix onto this plane. Define

$$
E^{*}(n, k)=\left\{(A, y, t) \in E(n, k) \times\left.\mathbf{R}|| y\right|^{2}+t^{2}=t\right\} .
$$

It can be checked that $E^{*}(n, k)$ is nonsingular and the projection $E^{*}(n, k) \rightarrow$ $G(n, k)$ is the $k$-sphere bundle obtained from $E(n, k)$ by compactifying each fiber to a sphere.

Lemma 2.7. Let $L \subset V \subset \mathbf{R}^{n}$ be nonsingular algebraic sets. Then there is a rational function $u: L \rightarrow G(n, k)$ where $u$ is the classifying map of the normal bundle $\nu(L, V)$ where $k=$ codimension of $L$ in $V$. In other words, $u(x)$ is the normal $k$-plane to $L$ which lies in the tangent plane of $V$ at $x$.

Proof. Let $r=$ codimension of $L$ in $\mathbf{R}^{n}$, and $s=$ codimension of $V$ in $\mathbf{R}^{n}$. It is known that the Gauss (classifying) maps $\alpha_{L}$ and $\alpha_{V}$ of the normal bundles of $L$ and $V$ in $\mathbf{R}^{n}$ are rational functions (see Lemma 2.3 of $\left[\mathbf{A K}_{2}\right]$ ). Denote $\left.\alpha_{V}\right|_{L}$ by $\alpha_{V}$

$$
\alpha_{L}: L \rightarrow G(n, r), \quad \alpha_{V}: L \rightarrow G(n, s) .
$$

Then $u: L \rightarrow G(n, k)$ is given by $u=\left(I-\alpha_{V}\right) \alpha_{L}$ (this formula says that first project on the plane $\alpha_{L}$ then project onto the complementary plane to $\alpha_{V}$ in $\alpha_{L}$ ), hence $u$ is rational.

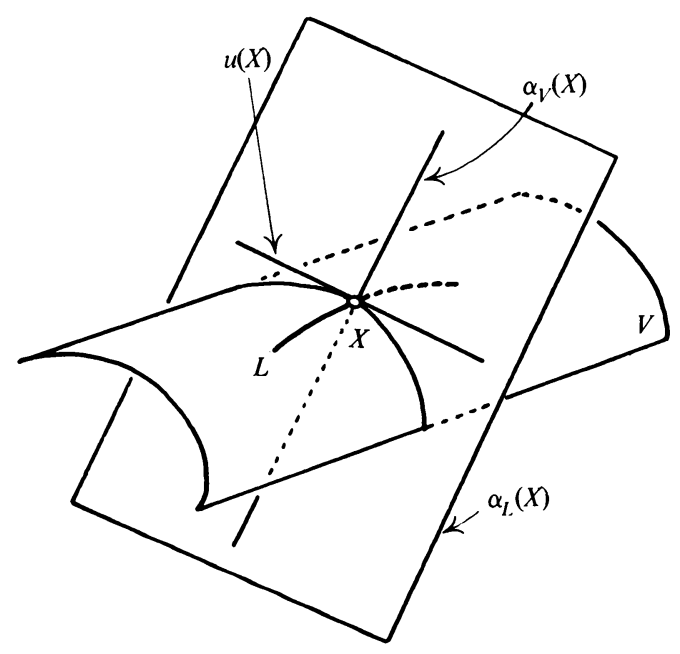

Figure 3 
Definition. An algebraic set $X$ has totally algebraic homology if for every $\alpha \in H_{k}(X ; \mathbf{Z} / 2 \mathrm{Z})$ there is a positive integer $n$ and a nonsingular projectively closed $k$-dimensional algebraic subset $V \subset X \times \mathbf{R}^{n}$ so that $\pi_{*}([V])=\alpha$ where $\pi: V \rightarrow X$ is the induced projection and $[V] \in H_{k}(V ; \mathbf{Z} / 2 \mathbf{Z})$ is the fundamental class of $V$.

REMARK. The Schubert cycles are algebraic subsets of $G(n, k)$ which generate the Z/2Z-homology. The Schubert cycles are sometimes singular; but by the following remark $G(n, k)$ has totally algebraic homology (for more details see Lemma 2.7 of $\left.\left[\mathbf{A K}_{\mathbf{2}}\right]\right)$.

In the above definition we can replace the requirements nonsingular and projectively closed by the requirement that $V$ be compact. This can be seen as follows. Let $V^{\prime}$ be the smallest projective algebraic set containing $V$. Then $V^{\prime}-V$ is a projective algebraic set, hence $\operatorname{dim}\left(V^{\prime}-V\right)<\operatorname{dim} V=k$. Therefore $V^{\prime}-V \subset$ Sing $V^{\prime}$. By resolving the singularities of $V^{\prime}[\mathbf{H}]$ we get a nonsingular $k$-dimensional projective algebraic set $W \subset \mathbf{R} P^{m}$ and a rational function $r: W \rightarrow V^{\prime}$ so that $r^{-1}$ (Nonsing $V^{\prime}$ ) is dense in $W$ and $r \mid r^{-1}$ (Nonsing $V^{\prime}$ ) is a diffeomorphism onto Nonsing $V^{\prime}$. Hence $r(W) \subset V$ and $r_{*}([W])=\left[V^{\prime}\right]$. Let $\theta: \mathbf{R} P^{m} \rightarrow G(m+1,1)$ be the canonical isomorphism, the Grassmann variety $G(m+1,1)$ is a subvariety of $\mathbf{R}^{(m+1)^{2}}$ then $Z=\{(r(x), \theta(x)) \in V \times G(m+1,1) \mid x \in W\}$ is a nonsingular projectively closed algebraic subset of $X \times \mathbf{R}^{n} \times \mathbf{R}^{(m+1)^{2}}$ which gives the same homology class as $[V]$.

LEMMA 2.8. An algebraic set $X$ has totally algebraic homology if and only if every element of $\eta_{*}(X)$ (unoriented bordism group of $X$ ) is represented by some $\pi: V \rightarrow X$ where $V$ is a nonsingular projectively closed algebraic subset of $X \times \mathbf{R}^{n}$ for some $n$ and $\pi$ is induced by projection.

Proof. Since $e v: \eta_{*}(X) \rightarrow H_{*}(X ; \mathbf{Z} / 2 \mathbf{Z})$ is onto where $e v(M \stackrel{f}{\rightarrow} X)=f_{*}[M]$, one way this proof is trivial. Now assume that $X$ has totally algebraic homology. It is known [CF] that $\eta_{*}(X)$ is generated by elements of the form

$$
U_{i} \times V_{j} \stackrel{\pi_{i j}}{\rightarrow} X
$$

with

$$
\pi_{i j}: U_{i} \times V_{j} \rightarrow V_{j} \stackrel{\alpha_{j}}{\rightarrow} X
$$

where $\left\{U_{i}\right\}$ generate $\eta_{*}$ (point) and $\left\{e v\left(\alpha_{j}\right)\right\}$ generate $H_{*}(X ; \mathbf{Z} / 2 Z)$. Hence we can assume that $V_{j}$ is a nonsingular algebraic subset of $X \times \mathbf{R}^{m}$ for some $m$ and $\alpha_{j}$ is induced by the projection. We can assume that $U_{i}$ are nonsingular algebraic sets in $\mathbf{R}^{n}$ [M]. Then $V_{j} \times U_{i} \subset X \times \mathbf{R}^{m} \times \mathbf{R}^{n}$ and the projection $\pi_{i j}: V_{j} \times U_{i} \rightarrow X$ generate $\eta_{*}(X)$ as a group. By translating we can make all $V_{i} \times U_{j}$ disjoint; since the group operation is disjoint union we are done.

CONJECTURE. Every compact smooth manifold is diffeomorphic to a nonsingular algebraic set with totally algebraic homology.

If the above conjecture were true, it would be an extremely useful fact. For instance it would allow the simpler proof of our main result (Theorem 2.10 ) in $\$ 3$. 
LeMMA 2.9. Let $X$ and $W$ be nonsingular algebraic sets, so $X$ has totally algebraic homology. Let $M$ be a closed smooth submanifold of $X$ and let $f: M \rightarrow W$ be a smooth map. Also let $L \subset M$ be an algebraic set, the germ of $M$ at $L$ be the germ of $a$ nonsingular algebraic set $V$, and the germ of $f$ at $L$ be the germ of an entire rational function $v: V \rightarrow W$. Then there is an $s$, an isotopy $h_{t}$ of $X \times \mathbf{R}^{s}$, an algebraic set $Z \subset X \times \mathbf{R}^{s}$, and an entire rational function $r: Z \rightarrow W$ so that

(1) $h_{1}(M \times 0)$ is a nonsingular component of $Z$,

(2) $h_{t}(x, 0)=(x, 0)$ for all $x \in L, t \in[0,1]$,

(3) $r(x, 0)=f(x)$ for all $x \in L$,

(4) $h_{t}$ can be as small as we want, and $\left.r h_{1}\right|_{M \times 0}$ can be as close an approximation to $f$ as we want (for some choice of $Z$ and $r$ ).

LEMMA 2.9'. Under the same hypothesis of Lemma 2.9 if in addition we assume that $W$ has totally algebraic homology then in the conclusion we get that $Z$ is a nonsingular algebraic set and $h_{1}(M \times 0)=Z$. Also along with (2), (3), (4) we get

(5) If $L$ is projectively closed then $Z$ is projectively closed.

Lemmas 2.9 and $2.9^{\prime}$ roughly say that if $X$ is a nonsingular algebraic set with totally algebraic homology (e.g. $\mathbf{R}^{n}$ ) then any closed smooth submanifold $M$ of $X$ can be made a nonsingular algebraic set in $X \times \mathbf{R}^{s}$ for some $s$; and also a smooth function from $M$ to a nice algebraic set may simultaneously be approximated by a rational function. We give a proof of Lemma 2.9; the proof of Lemma 2.9' is similar but somewhat harder than this proof. See Proposition 2.8 of $\left[\mathbf{A K} \mathbf{K}_{2}\right]$ for the proof of Lemma 2.9'.

Proof of Lemma 2.9. Let $X$ and $W$ be nonsingular algebraic subsets of $\mathbf{R}^{n}$ and $\mathbf{R}^{m}$ respectively. Let $T$ be a tubular neighborhood of $M$ in $X$; and let $g: T \rightarrow$ $E(n, k)$ be the classifying map of the normal bundle of $M$ in $X$, where $k$ is the codimension of $M$ in $X$. Then $g$ has the properties

(a) $g$ is transverse to $G(n, k)$,

(b) $g^{-1}(G(n, k))=M$.

$E(n, k)$ is a nonsingular algebraic set in $\mathbf{R}^{q}$ for some $q$, and $G(n, k)$ is a nonsingular algebraic subset of $E(n, k)$.

Let $N$ be a neighborhood of $L$ in $M$; by Lemma $\left.2.7 g\right|_{N}$ equals a rational function $u$. We can extend $f$ to $T$ by composing $f$ with projection from $T$ to $M$; also recall that $\left.f\right|_{N}$ equals a rational function $v$. By Lemma 2.5 we can approximate $(f-v) \times(g-u)$ by a polynomial $\phi^{\prime}:\left(\mathbf{R}^{n}, L\right) \rightarrow\left(\mathbf{R}^{m} \times \mathbf{R}^{q}, 0\right)$ near $M$. Let $\phi=\phi^{\prime}$ $+(v, u)$; then $\phi$ approximates $f \times g$ near $M$. Also $\left.\phi\right|_{L}=v \times u$.

Now define $S=\left\{(x, y) \in X \times \mathbf{R}^{m+q} \mid \phi(x)+y \in W \times E(n, k), y\right.$ is normal to $W \times E(n, k)$ at $\phi(x)+y$ in $\left.\mathbf{R}^{m+q}\right\} . S$ is an algebraic set. To see this, let $\rho:$ $\mathbf{R}^{m+q} \rightarrow \mathbf{R}$ be a polynomial with $\rho^{-1}(0)=W \times E(n, k)$, and by Lemma 2.7 there is a rational function $\delta: W \times E(n, k) \rightarrow G(m+q, r), r=$ codimension of $W \times$ $E(n, k)$, which is the normal bundle map of $W \times E(n, k) \subset \mathbf{R}^{m+q}$. By considering the elements of $G(m+q, r)$ as $(m+q) \times(m+q)$ projection matrices onto $r$ planes, we can write $S=\left\{(x, y) \in X \times \mathbf{R}^{m+q} \mid \rho(\phi(x)+y)=0, \delta(\phi(x)+y) y=\right.$ $y$ ) i.e. $S$ is an algebraic subset of $X \times \mathbf{R}^{m+q}$. 

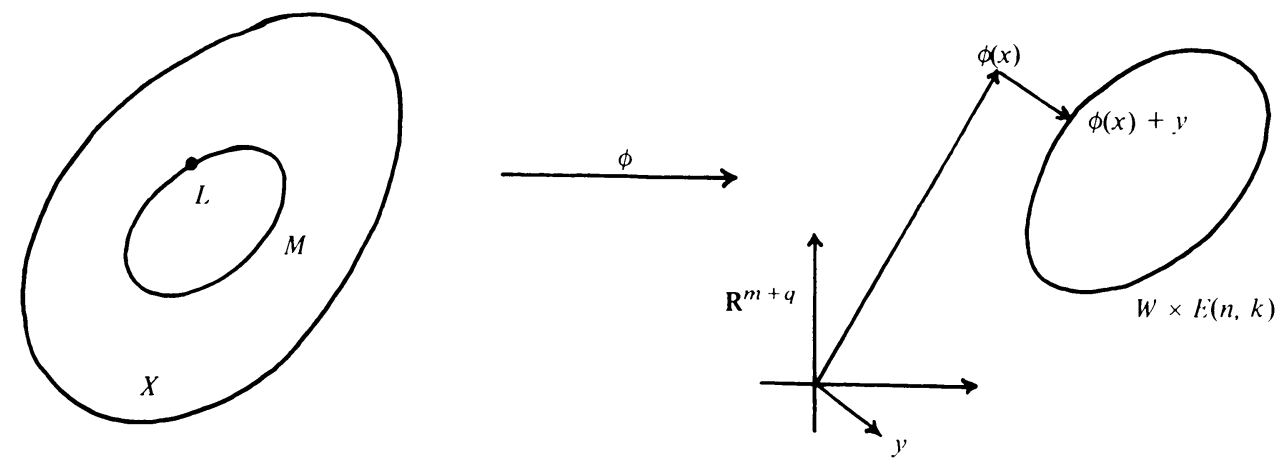

FIGURE 4

Let $w: S \rightarrow W \times E(n, k)$ be the rational function $w(x, y)=\phi(x)+y$. Since for every $x \in T, \phi(x)$ is close to $W \times E(n, k)$ there is a unique $y(x)$ so that $\phi(x)+$ $y(x)$ is the unique closest point to $\phi(x)$ in $W \times E(n, k)$ (see Figure 4). Now define $J=\left\{(x, y(x)) \in S \cap\left(T \times \mathbf{R}^{m+q}\right)\right\}$ then we have:

(A) $J$ is an open subset of Nonsing $(S)$.

(B) The function $x \rightarrow w(x, y(x))$ is close to $f \times g$ on $T$ (since $y(x)$ is small).

(C) $w(x, 0)=(v(x), u(x))=(f(x), g(x))$ for all $x \in L$.

Hence by transversality $(f \times g)^{-1}(W \times G(n, k))=g^{-1}(G(n, k))=M$ is isotopic to $\{x \in T \mid w(x, y(x)) \in W \times G(n, k)\}$ fixing $L$, which is in turn isotopic to $J \cap w^{-1}(W \times G(n, k))$ fixing $L \times 0$. Let

$$
\hat{Z}=S \cap w^{-1}(W \times G(n, k)) \quad \text { and } \quad Z^{\prime}=J \cap w^{-1}(W \times G(n, k)) .
$$

Then by Lemma 2.2 all the points of $Z^{\prime}$ are nonsingular of dimension $\operatorname{dim} M$ in $\hat{Z}$. Let $Z=\operatorname{Sing}(\operatorname{Sing}(\ldots(\operatorname{Sing} \hat{Z}) \ldots))$ where we take Sing a number of times so that $\operatorname{dim} Z=\operatorname{dim} M$. Then notice that $Z$ is an algebraic subset of $X \times \mathbf{R}^{m+q}$ and $Z^{\prime}$ is a nonsingular component of $Z$. We have constructed an isotopy $h_{t}$ of $M \times 0$ to $Z^{\prime}$ which fixes $L \times 0$. To construct the rational function $r$, let $\pi: \mathbf{R}^{m} \times \mathbf{R}^{q} \rightarrow \mathbf{R}^{m}$ be the projection then let $r=\pi \circ w$. $r$ has the required properties.

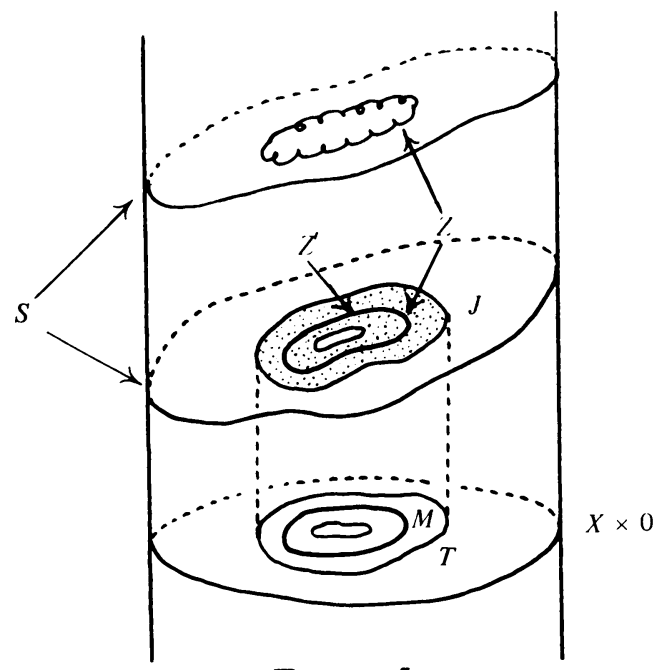

Figure 5 
TheORem 2.10 (MAIN TheOREM). Let $M$ be a closed smooth submanifold of $a$ nonsingular algebraic set $V$ with totally algebraic homology. Let $M_{i}, i=1,2, \ldots, k$, be closed smooth submanifolds of $M$ in general position. Then there is an arbitrarily small isotopy of $V \times \mathbf{R}^{n}$ for some $n$, which simultaneously takes $M \times 0$ and all $M_{i} \times 0$ to nonsingular projectively closed algebraic sets.

REMARK. By letting $V=\mathbf{R}^{m}$ we get Theorem 1.1 as a corollary to this theorem.

COROllaRY 2.11. The interior of a compact smooth manifold $M$ with $\partial M \neq \varnothing$, is diffeomorphic to a nonsingular algebraic set $V$ which is properly imbedded in $\mathbf{R}^{n}$ for some $n$.

Proof. By $\left[\mathbf{A K}_{\mathbf{2}}\right]$ or $\left[\mathbf{A K}_{3}\right]$ we can find closed smooth submanifolds $M_{i}, i=$ $1, \ldots, k$, of a manifold $M^{\prime}$ in general position with $\partial M^{\prime}=\partial M$ and $M^{\prime} / \cup M_{i} \simeq$ cone $\left(\partial M^{\prime}\right)$. Let $W=M \cup_{\partial} M^{\prime}$. By Theorem 2.10 we can assume that $W$ is a nonsingular algebraic set and all $M_{i}$ are nonsingular algebraic subsets in some $\mathbf{R}^{p}$. Let $f(x)$ and $g(x)$ be polynomials with $f^{-1}(0)=W, g^{-1}(0)=\cup M_{i}$. Let $F(x, t)=$ $f(x)^{2}+(\operatorname{tg}(x)-1)^{2}$. Then let $F^{-1}(0)=V \subset \mathbf{R}^{n}, n=p+1$. The algebraic set $V$ has the required properties.
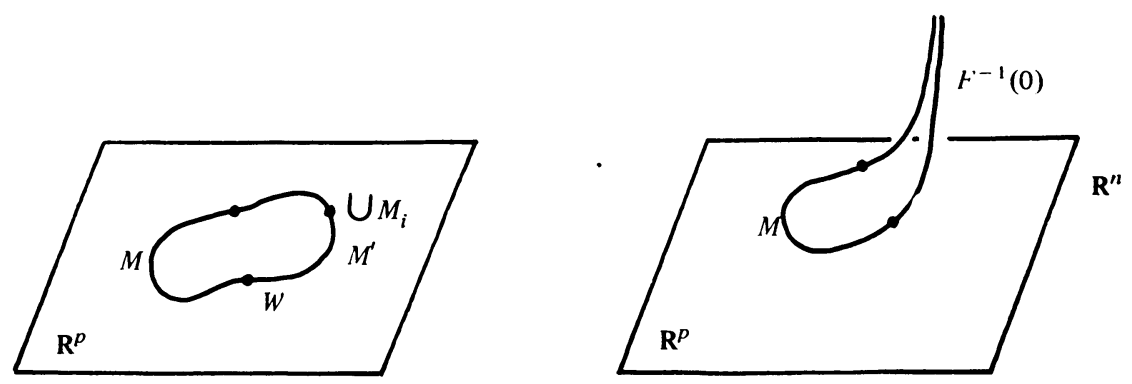

Figure 6

3. A quick proof of a special case. We can try to prove Theorem 2.10 by first assuming $M$ to be a nonsingular algebraic subset of $V \times \mathbf{R}^{m}$ for some $m$ (by Lemma 2.9') and then isotoping the submanifolds $M_{i}$ to nonsingular algebraic subsets of $M$. This seems to be very difficult at the present time; but if we assume that $M$ has totally algebraic homology then we can achieve this Theorem 3.1. Theorem 3.1 has also a somewhat stronger conclusion than Theorem 2.10 , also in Theorem 3.1 $M$ can be noncompact.

THEOREM 3.1. Let $M$ be a nonsingular algebraic set with totally algebraic homology, and let $M_{i}, i=1, \ldots, k$, be closed smooth submanifolds of $M$ in general position. Then there is an arbitrarily small isotopy of $M \times \mathbf{R}^{n}$ for some $n$, which simultaneously takes $M \times 0$ and all $M_{i} \times 0$ to nonsingular algebraic sets. In addition, this isotopy takes $M \times 0$ to an algebraic set with totally algebraic homology.

Proof. We prove this by induction on $k$. Assume that $M_{1}, M_{2}, \ldots, M_{k-1}$ are already nonsingular algebraic subsets of $M$. Since $M$ has totally algebraic 
homology by Lemma $2.9^{\prime} M_{k}$ is isotopic to a nonsingular algebraic subset $Z_{k}$ of $M \times \mathbf{R}^{n}$ by an arbitrarily small isotopy, for some $n$. By extending this isotopy we can assume that it takes $M \times 0$ to some manifold $M^{\prime}$ in $M \times \mathbf{R}^{n}$ such that $M^{\prime}-M \times 0$ has compact closure and the composition $M^{\prime} \hookrightarrow M \times \mathbf{R}^{n} \rightarrow M$ is a diffeomorphism. (In particular $Z_{k} \subset M^{\prime}$.)

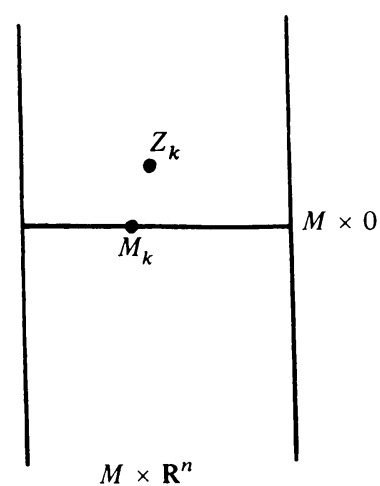

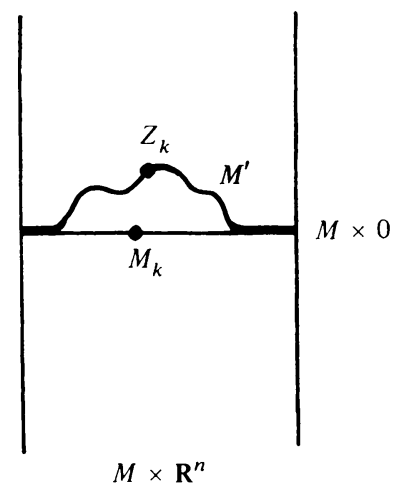

FigURE 7

By Lemma 2.4 we may pick a proper polynomial $\rho: M \times \mathbf{R}^{n} \rightarrow \mathbf{R}$ so that $\rho^{-1}(0)=Z_{k}$. Let $\alpha: M \rightarrow \mathbf{R}^{n}$ be a smooth function with compact support so that $M^{\prime}=\left\{(x, y) \in M \times \mathbf{R}^{n} \mid y=\alpha(x)\right\}$. Pick a smooth function $\beta: M \times \mathbf{R}^{n} \rightarrow[0,1]$ with compact support with $\beta(x, y)=1$ when $|y|<|\alpha(x)|$. We now define $\gamma$ : $M \times \mathbf{R}^{n} \rightarrow \mathbf{R}^{n}$ :

$$
\gamma(x, y)=\beta(x, y)(y-\alpha(x))+(1-\beta(x, y)) \rho^{2}(x, y) y .
$$

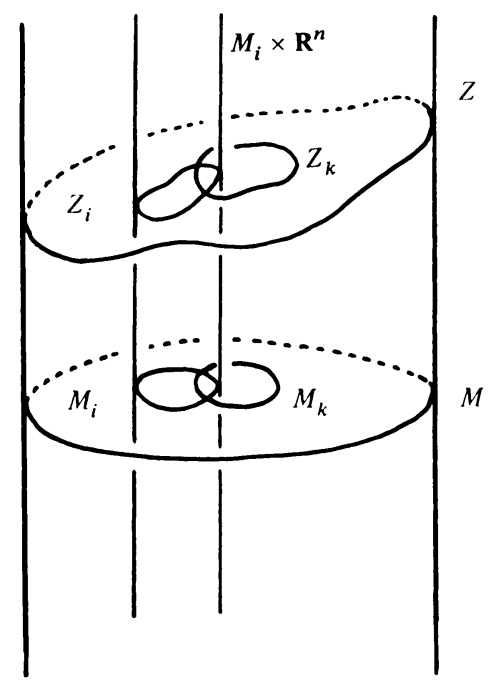

FIGURE 8 
By inspection $\gamma^{-1}(0)=\left\{(x, y) \in M \times \mathbf{R}^{n} \mid y=\alpha(x)\right\}=M^{\prime}$ and $\gamma$ equals the polynomial $\rho^{2}(x, y) y$ outside of a compact set. By Lemma 2.5 we can $C^{1}$ approximate $\gamma(x, y)-\rho^{2}(x, y) y$ by an entire rational function $u:\left(M \times \mathbf{R}^{n}, Z_{k}\right) \rightarrow\left(\mathbf{R}^{n}, 0\right)$. This approximation is close on all $M \times \mathbf{R}^{n}$, not just on a compact set. Hence if we let $w(x, y)=u(x, y)+\rho^{2}(x, y) y$, then $w$ is $C^{1}$ close to $\gamma$ on $M \times \mathbf{R}^{n}$. Then the properness of $\rho(x, y)$ insures that $w^{-1}(0)$ is isotopic to $\gamma^{-1}(0)=M^{\prime}$ by an arbitrarily small isotopy fixing $Z_{k}$. In particular the nonsingular algebraic set $Z=w^{-1}(0)$ contains $Z_{k}$. Let $Z_{i}=Z \cap\left(M_{i} \times \mathbf{R}^{n}\right), i=1, \ldots, k-1$. By transversality each $Z_{i}$ is a nonsingular algebraic subset of $Z$ which is isotopic to $M_{i}$.

By Lemma 2.1 we have an isotopy of $M$ carrying $M_{k}$ to $M_{k}^{\prime}$ and fixing $M_{i}$ for $i<k$, where $M_{k}^{\prime}$ is the image of $Z_{k}$ under the projection $Z_{k} \subset M \times \mathbf{R}^{n} \rightarrow M$. We follow this isotopy in the $\mathbf{R}^{n}$ coordinate to isotop $\left(M,\left\{M_{i}\right\}\right)$ to $\left(Z,\left\{Z_{i}\right\}\right)$.

To finish the theorem we must prove that $Z$ has totally algebraic homology. Take any $\alpha \in H_{i}(Z ; Z / 2 Z)$. Let $h: Z \rightarrow M$ be the diffeomorphism induced by projection. Pick an $i$-dimensional nonsingular algebraic set $U \subset M \times \mathbf{R}^{k}$ so that $\pi_{*}([U])=h_{*}(\alpha)$, where $\pi: U \rightarrow M$ is induced by projection $M \times \mathbf{R}^{k} \rightarrow M$ and $[U]$ is the fundamental class of $U$. Let $V \subset Z \times \mathbf{R}^{k}$ be the pullback $V=\{(x, y, t) \in$ $\left.M \times \mathbf{R}^{n} \times \mathbf{R}^{k} \mid(x, y) \in Z,(x, t) \in U\right\}$. Then $V$ is diffeomorphic to $U$ and $\pi_{*}^{\prime}([V])$ $=\alpha$ where $\pi^{\prime}: V \rightarrow Z$ is induced by the projection $M \times \mathbf{R}^{n} \times \mathbf{R}^{k} \rightarrow M \times \mathbf{R}^{n}$. Hence $Z$ has totally algebraic homology.

4. Proof of Main Theorem. The following lemma basically says that for any given closed smooth manifold $M$ and transversally intersecting closed smooth submanifolds $M_{i}, i=1, \ldots, k$, then there is a nonsingular algebraic set $Z$ and transversally intersecting nonsingular algebraic subsets $Z_{i}, i=1, \ldots, k$, such that $\left(M ; M_{1}, \ldots, M_{k}\right)$ is relatively cobordant to $\left(Z ; Z_{1}, \ldots, Z_{k}\right)$. In other words, this lemma proves Theorem 2.10 up to cobordism. Then the proof of Theorem 2.10 is done by showing this "cobordism" implies the "isotopy". The idea of the proof of this lemma is similar to that of $[\mathbf{A}]$, where it was shown that for some P.L. manifolds $M(M$, the nonsmooth points of $M)$ is relatively cobordant to (an algebraic set $V$, Sing $V$ ).

Lemma 4.1. Let $M$ be a closed smooth manifold, and let $M_{i}, i=1,2, \ldots, k$, be closed smooth submanifolds of $M$ in general position. Let $\varphi$ be a map $\varphi: M \rightarrow V$ where $V$ is a nonsingular algebraic set with totally algebraic homology. Then there is a smooth manifold $T$ with submanifolds $T_{i}$ in general position, and a map $\psi: T \rightarrow V$ with the following properties:

(1) $\partial T \cap T_{i}=\partial T_{i}$.

(2) $\partial T$ is the disjoint union $M \cup \cup_{I} U^{I}$ where the $U^{I}$ are projectively closed nonsingular algebraic subsets of $V \times R^{n}$ for some $n$ and $I$ runs over all subsets of $\{1,2, \ldots, k\}$.

(3) $\left.\psi\right|_{M}=\varphi$ and $\left.\psi\right|_{U^{\prime}}$ is induced by projection $V \times \mathbf{R}^{n} \rightarrow V$.

(4) $T_{i} \cap M=M_{i}$.

(5) $\cap_{i \in J} T_{i} \cap U^{I}$ is empty if $J \not \subset I$ and is a nonsingular algebraic set $U_{J}^{I}$ if $J \subset I$. 
(6) Let $c_{i}$ by the codimension of $M_{i}$ in $M$. Then there is a large $m$ and entire rational functions $\lambda_{i}^{I}: U_{I}^{I} \rightarrow G\left(m, c_{i}\right)$ so that for $J \subset I \quad U_{J}^{I}=\left\{(x, y) \in U_{I}^{I} \times\right.$ $R^{(m+1) k} \mid\left(\lambda_{i}^{I}(x), y_{i}\right) \in E^{*}\left(m, c_{i}\right)$ if $i \in I-J$, and $y_{i}=0$ if $\left.i \notin I-J\right\}$. (We think of $R^{(m+1) k}$ as $(m+1) \times k$ matrices then $y_{i}$ is the ith column vector of $y$.) Thus $U^{I}=U_{\phi}^{I}$ is the total space of a $c_{i}$ sphere bundle over $U^{I} \cap T_{i}=U_{i}^{I}$ for each $i \in I$. Notice that we may extend each $\lambda_{i}^{I}$ to $U_{i}^{I}$ by composing with projection of $U_{i}^{I}$ to $U_{I}^{I}$. This $\lambda_{i}{ }^{I}$ classifies the normal bundle of $U_{i}^{I}$ in $U^{I}$.

(7) $U^{I}$ is empty if $\cap_{i \in I} M_{i}$ is empty.

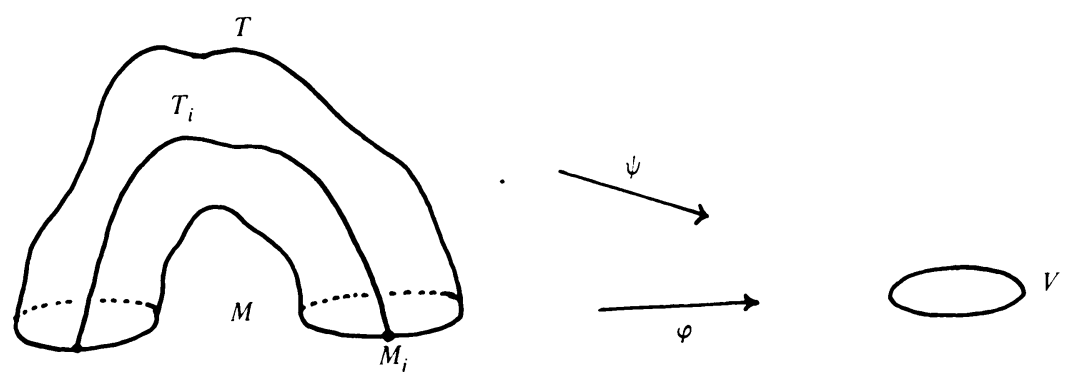

Figure 9

Proof. After the end of this proof we give a few examples of the construction of $T$ and the $T_{i}$ 's.

For any $I \subset\{1, \ldots, k\}$ let $M_{I}=\bigcap_{i \in I} M_{i}$. By convention, $M_{\phi}=M$.

We will prove this by induction on the number of subsets $I$ of $\{1, \ldots, k\}$ so that $M_{I}$ is nonempty. To start the induction, if $M_{I}$ is empty for all subsets then $M=M_{\phi}$ is empty so the claim is true, letting $T$ be the empty manifold.

Now take any $M$ and $M_{i}$ as above. Pick a subset $I_{0}$ of $\{1, \ldots, k\}$ so that $M_{I_{0}}$ is nonempty but $M_{I}$ is empty for all $I \supsetneqq I_{0}$. Denote $L=M_{I_{0}}$. Pick $m$ large enough that $G(m, c)$ is a classifying space for all $c$ bundles over manifolds of dimension $\leqslant \operatorname{dim} M-c$. For each $i$ we have a map $\alpha_{i}: M_{i} \rightarrow G\left(m, c_{i}\right)$ classifying the normal bundle of $M_{i}$ in $M$. We have a map $\beta: L \rightarrow V \times \prod_{i \in I_{0}} G\left(m, c_{i}\right)$ which is $\left.\varphi\right|_{L}$ to $V$ and $\left.\alpha_{i}\right|_{L}$ to $G\left(m, c_{i}\right)$. By Lemma 2.8 there is a manifold $K$ and a map $\gamma$ : $K \rightarrow V \times \Pi_{I_{0}} G\left(m, c_{i}\right)$ so that $\partial K$ is the disjoint union of $L$ and a projectively closed nonsingular algebraic set $U^{\prime} \subset V \times \Pi_{I_{0}} G\left(m, c_{i}\right) \times R^{a},\left.\gamma\right|_{L}=\beta$ and $\left.\gamma\right|_{U^{\prime}}$ is the obvious projection. Define $\mu_{j}: V \times \Pi G\left(m, c_{i}\right) \rightarrow G\left(m, c_{j}\right)$ to be projection. Define manifolds $S_{I}$ by letting $S_{I}$ be empty if $I \not I_{0}$, and if $I \subset I_{0}$

$$
\begin{aligned}
S_{I}=\left\{(x, y) \in K \times R^{(m+1) k} \mid\right. & \left(\mu_{i} \gamma(x), y_{i}\right) \in E^{*}\left(m, c_{i}\right) \\
& \text { if } \left.i \in I_{0}-I, \text { and } y_{i}=0 \text { if } i \notin I_{0}-I\right\}
\end{aligned}
$$

where $y_{i} \in \mathbf{R}^{m+1}$ is the $i$ th coordinate of $y$. Since $\left.\mu_{i} \gamma\right|_{L}=\left.\alpha_{i}\right|_{L}$ there is a diffeomorphism $h: A \rightarrow B$ where $A$ is a closed tubular neighborhood of $L \times 0$ in $\partial S_{\phi}$ and $B$ is a tubular neighborhood of $L$ in $M$. Furthermore, $h\left(A \cap S_{i}\right)=B \cap M_{i}$ for each $i \in I_{0}$ and $B \cap M_{i}$ is empty for each $i \notin I_{0}$. We now form a manifold $S^{\prime}=S_{\phi} \cup$ $M \times[0,1]$ where we identify $A \subset \partial S_{\phi}$ with $B \times 1 \subset M \times[0,1]$ via $h$. Let $S_{I}^{\prime}=S_{I}$ $\cup M_{I} \times[0,1] \subset S^{\prime}$. Then the $S_{i}^{\prime}$ are in general position in $S^{\prime}$ and $\bigcap_{i \in I} S_{i}^{\prime}=S_{I}^{\prime}$. We may define a map $\theta: S^{\prime} \rightarrow V$ by first retracting $S^{\prime}$ to $S_{\phi} \cup L \times[0,1] \cup M \times 0$ 
and then mapping $S_{\phi}$ by projecting to $K$, mapping by $\gamma$ to $V \times \Pi G\left(m, c_{i}\right)$ and projecting to $V$. The map on $L \times[0,1] \cup M \times 0$ will be projection to $M$ followed by $\varphi$.

Notice that $\left.\theta\right|_{M \times 0}=\varphi$. Also if we denote $U^{I_{0}}=S_{\phi} \cap U^{\prime} \times R^{(m+1) k}$ then $\left.\theta\right|_{U^{\prime} 0}$ is projection. In addition $\partial S^{\prime}$ is the disjoint union of $M \times 0, U^{I_{0}}$ and $M^{\prime}=\left(S_{\phi} \cap\right.$ $\left.L \times R^{(m+1) k}-\AA\right) \cup(M-\not \circ) \times 1$. Let $M_{I}^{\prime}=M^{\prime} \cap S_{I}^{\prime}$. Then $M_{I}^{\prime}$ is empty if $M_{I}$ is. Also $M_{I_{0}}^{\prime}$ is empty. So by induction there are $T^{\prime}, T_{i}^{\prime}$, and $\psi^{\prime}: T^{\prime} \rightarrow V$ satisfying (1)-(6) (with everything primed and $\left.\psi^{\prime}\right|_{M^{\prime}}=\theta_{M^{\prime}}$ ). Let $T=S^{\prime} \cup T^{\prime}, T_{i}=S_{i}^{\prime} \cup T_{i}^{\prime}$ and $\psi=\psi^{\prime} \cup \theta$ then we are done.

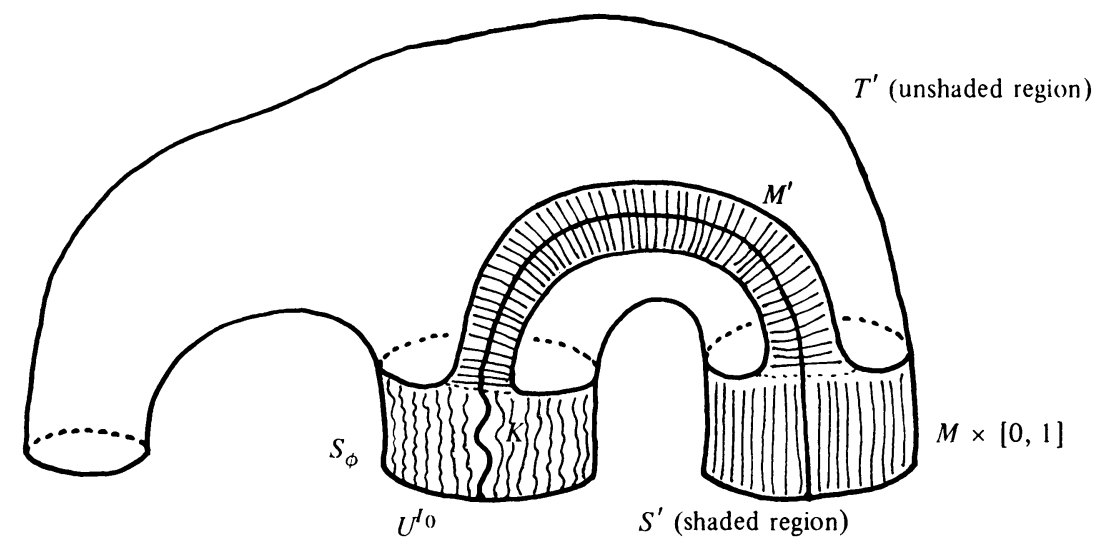

Figure 10

As promised, we now give examples of the $T$ and $T_{i}$ 's constructed in the above proof. We let $V$ be a point so we do not have to mention the maps $\varphi$ or $\psi$.

Example A. $M=S^{1}, k=1, M_{1}$ is a point. Then $L=M_{1}$ and $K$ can be an interval. $U^{1}$ is a circle and $U_{1}^{1}$ is a point. Figure 11(a) below shows $S^{\prime}$. Notice that $M^{\prime} \cap S_{1}^{\prime}$ is empty. Now since $M^{\prime}$ bounds we may let $U^{\phi}$ be empty, then Figure 11(b) shows $T$.
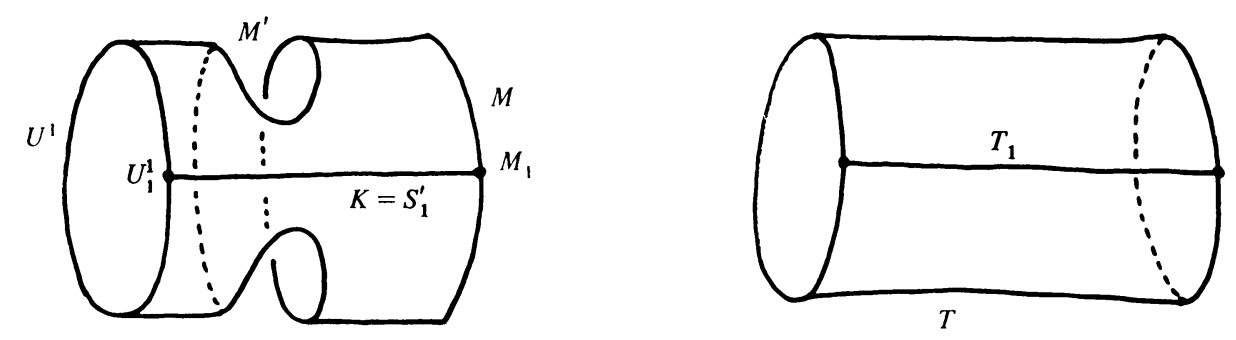

Figure 11

ExAmple B. Let $M=R P^{2}$ and let $M_{1}$ and $M_{2}$ be linear subspaces $M_{i}=$ $\left\{\left(x_{0}: x_{1}: x_{2}\right) \mid x_{i}=0\right\}$. Then $M_{12}$ is a point. We let $k$ be an interval. Then $U^{12}$ is a torus $S^{1} \times S^{1}$ and $U_{1}^{12}=S^{1} \times b$ and $U_{2}^{12}=a \times S^{1} . M^{\prime}$ is the connected sum of a torus and a projective plane. (In fact, $M^{\prime}$ is the torus $S^{1} \times S^{1}$ blown up at the point $(a, b)$, the $M_{i}^{\prime}$ are the blowups of the $S^{1} \times b$ and $a \times S^{1}$ and are disjoint.) 
By representing $M^{\prime}$ as a hexagon with sides identified we indicate $M_{1}^{\prime}$ and $M_{2}^{\prime}$ in Figure 12. Now the normal bundle of each $M_{i}^{\prime}$ in $M^{\prime}$ is nontrivial, hence we let $U^{i}$ be the nontrivial $S^{1}$ bundle over $S^{1}$, i.e., the $U^{i}$ are Klein bottles and the $U_{i}^{i}$ are circles, the zero sections of these bundles. After glueing these on we are left with an $M^{\prime \prime}$ which is in fact $S^{1} \times S^{1} \# R P^{2}$. Also the $M_{i}^{\prime \prime}$ are all empty. We cobord the $M^{\prime \prime}$ to $R P^{2}$, say and let $U^{\phi}=R P^{2}$.

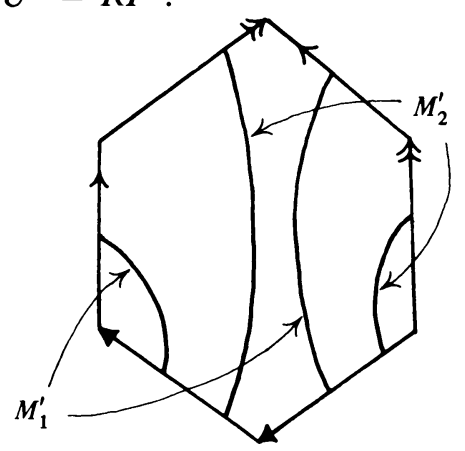

FIGURE 12

EXAMPLE C. $M=S^{2}, M_{1}$ and $M_{2}$ are two great circles and $M_{3}$ is a point in $S^{2}-\left(M_{1} \cup M_{2}\right)$. Then $U^{123}, U^{13}$ and $U^{23}$ must be empty. We may let $U^{3}$ be a two sphere and $U_{3}^{3}$ a point in $U^{3} . S^{\prime}$ is a solid disc with two holes and $M^{\prime}$ is a two sphere and $M_{1}^{\prime}$ and $M_{2}^{\prime}$ are circles (see Figure 13). Since $M_{1}^{\prime} \cap M_{2}^{\prime}$ is two points, it bounds so we may make $U^{12}$ empty. We thus add an $S^{1} \times S^{1} \times[-1,1]$ to our cobordism. Our new $M^{\prime \prime}$ is the connected sum of two tori and $M_{1}^{\prime \prime}$ and $M_{2}^{\prime \prime}$ are as in Figure 14.
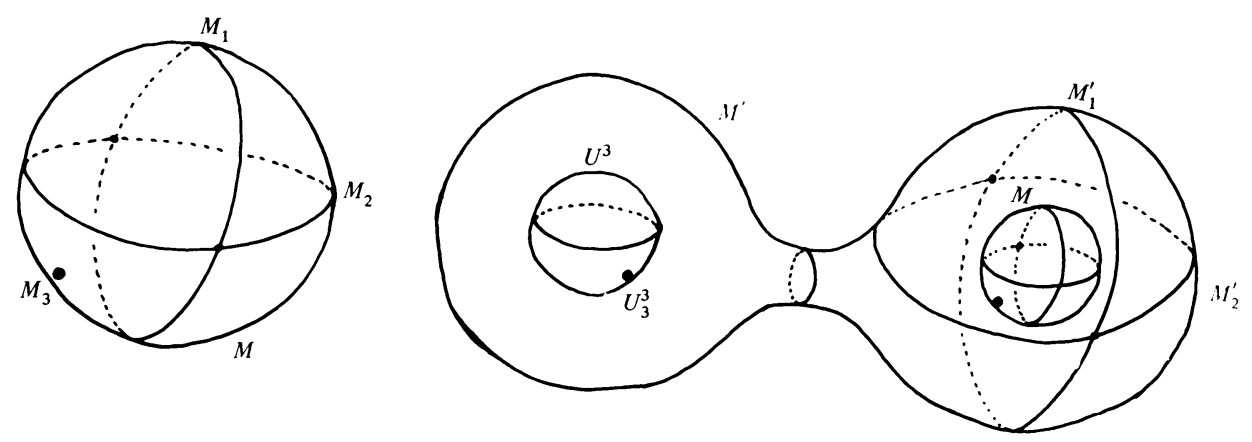

FIGURE 13

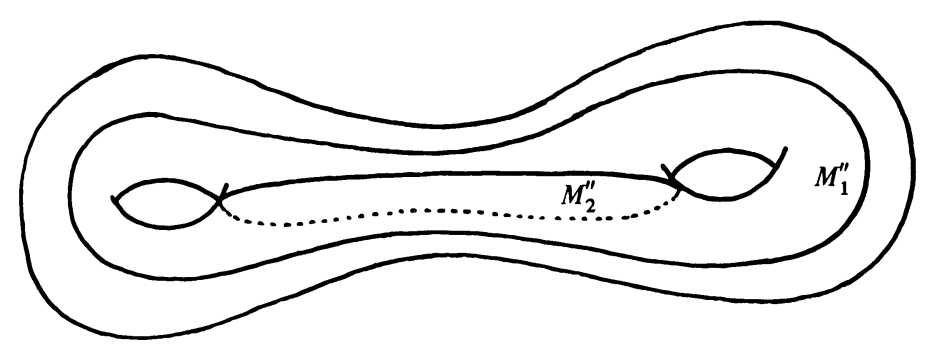

Figure 14 
Since $M_{1}^{\prime \prime}$ and $M_{2}^{\prime \prime}$ bound we may make $U^{1}$ and $U^{2}$ be empty. We are then left with a surface of genus 2 for $M^{\prime \prime \prime}$ which bounds so we may let $U^{\phi}$ be empty also. The actual $T$ we have constructed is rather hard to describe, it is the union of two $S^{2} \times[0,1]$ 's, an $S^{1} \times S^{1} \times[0,1]$, two $D^{2} \times S^{1}$ 's and a manifold with boundary $S^{1} \times S^{1} \# S^{1} \times S^{1}$. The $T_{i}$ 's are easier to describe. $T_{3}$ is an interval. $T_{1}$ and $T_{2}$ are each a torus minus a disc. $T_{1} \cap T_{2}$ is an interval (see Figure 15).

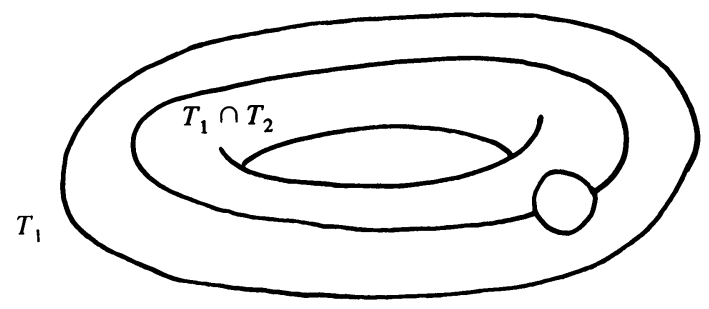

FIGURE 15

Proof OF TheOrem 2.10. Before proceeding with a detailed proof, we give a rough sketch to guide the reader. Using Lemma 4.1 we find a closed smooth submanifold $N \subset V \times \mathbf{R}^{n}$ for some $n$ ( $N$ is essentially the double of $T$ of Lemma 4.1), and submanifolds $N_{i} \subset N, i=1, \ldots, k$, with the properties that:

(1) $M$ is a smooth submanifold of $N$.

(2) There is a projectively closed nonsingular algebraic set $U \subset V \times \mathbf{R}^{n}$ with $U \subset N$ and disjoint from $M$.

(3) $M \cup U$ is a codimension 1 submanifold of $N$, which compactly separates $N$.

(4) $N_{i} \cap M=M_{i}$.

(5) $N_{i} \cap U$ is a nonsingular algebraic set. There is also a technical condition which will appear in the proof, roughly it says that for each $i$ the connected components of $U$ which intersect $N_{i}$ are the total space of an "algebraic" sphere bundle over $N_{i} \cap U$.

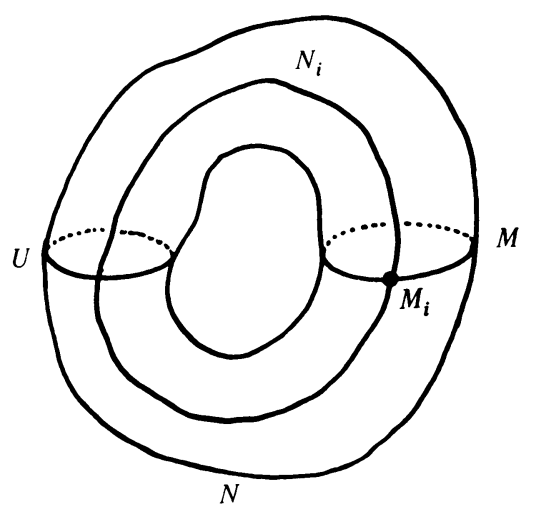

Figure 16 
We then proceed to make $N, M$ and $N_{i}$ 's algebraic. We show that for each $i$ there are algebraic sets $Y_{i}$ and $Y_{i}^{\prime}$ so that the pair $\left(N, N_{i}\right)$ is diffeomorphic to $\left(Y_{i}, Y_{i}^{\prime}\right)$ by a diffeomorphism $\sigma_{i}$ so that $\left.\sigma_{i}\right|_{U}$ is an entire rational function. By Lemma 2.9 we may isotop $N$ to a nonsingular component $Z^{\prime}$ of an algebraic set $Z$ fixing $U$ so that all the above diffeomorphisms to $\left(Y_{i}, Y_{i}^{\prime}\right)$ are entire rational functions. Hence $N_{i}$ have become algebraic by Lemma 2.2. Now by Lemmas 2.6 and 2.3 we can isotop $M$ to a nonsingular algebraic subset $X$ of $Z^{\prime}$. Hence each $M_{i}$ is isotopic to the nonsingular algebraic set $X_{i}=N_{i} \cap X$.

We now proceed with the proof: Let $\varphi: M \rightarrow V$ be the inclusion. Let $T$ be the cobordism obtained in Lemma 4.1. Then by making sure $n$ is big enough, we get an imbedding $\eta: T \rightarrow V \times R^{n}$ so that $\eta(x)=(x, 0)$ for $x \in M$ and $\left.\eta\right|_{U^{\prime}}$ is inclusion for each $I$. (It might be necessary to translate the $U^{I}$ 's a bit to make them disjoint.)

We now let $N$ be the boundary of $\eta(T) \times[-1,1] \subset\left(V \times R^{n}\right) \times R$ after smoothing out the corners. Also $N_{i} \subset N$ are the boundaries of $\eta\left(T_{i}\right) \times[-1,1]$, $i=1, \ldots, k$. Thus $N$ is the double of $T$ and the $N_{i}$ are the doubles of $T_{i}$. Denote $U=\cup_{I\{1, \ldots, k\}} U^{I} \times 0$. Then $M \times 0 \times 0, U$ and the $N_{i}$ 's are in general position in $N, M \times 0 \times 0 \cup U$ compactly separates $N$ and the germ of $N$ at $U$ is the germ of the nonsingular algebraic set $\cup_{I} U^{I} \times R$ at $U$.

We now construct the $Y_{i}$ and $Y_{i}^{\prime}$. Pick $\beta_{i}: N_{i} \rightarrow G\left(m, c_{i}\right)$ classifying the normal bundle of $N_{i}$ in $N$. We may require that $\left.\beta_{i}\right|_{N_{i} \cap U}$ be an entire rational function and that in fact $\left.\beta_{i}\right|_{N_{i} \cap U^{I}}=\left.\beta_{i}\right|_{U_{i}^{\prime}}$ is the composition of projection to $U_{I}^{I}$ and $\lambda_{i}^{I}$ if $i \in I$ (see Lemma 4.1). By Lemma 2.9' there is a nonsingular algebraic set $Y_{i}^{\prime} \subset V \times R^{n}$ $\times R \times R^{a}$, an entire rational function $\gamma_{i}: Y_{i}^{\prime} \rightarrow G\left(m, c_{i}\right)$ and a diffeomorphism $h_{i}$ : $N_{i} \rightarrow Y_{i}^{\prime}$ so that $h_{i}(x)=(x, 0)$ and $\gamma_{i}(x, 0)=\beta_{i}(x)$ for all $x \in N_{i} \cap U$. In addition, $\gamma_{i} h_{i}$ approximates $\beta_{i}$, hence $\gamma_{i} h_{i}$ and $\beta_{i}$ are homotopic. Define $Y_{i}^{\prime \prime}=\left\{(x, y) \in Y_{i} \times\right.$ $\left.R^{m+1} \mid\left(\gamma_{i}(x), y\right) \in E^{*}\left(m, c_{i}\right)\right\}$. Then since $\gamma_{i} h_{i}$ and $\beta_{i}$ are homotopic, the normal bundle of $Y_{i}^{\prime}$ in $Y_{i}^{\prime \prime}$ is equivalent to the normal bundle of $N_{i}$ in $N$. Hence there is an imbedding $\sigma_{i}: N \rightarrow V \times R^{n} \times R \times R^{a} \times R^{m+1}$ so that $\left.\sigma_{i}\right|_{U}$ is an entire rational function, $\sigma_{i}(U) \subset Y_{i}^{\prime \prime},\left.\sigma_{i}\right|_{N_{i}}=h_{i}$ and the germ of $\sigma_{i}(N)$ at $\sigma_{i}\left(U \cup N_{i}\right)$ is the germ of $Y_{i}^{\prime \prime}$ at $\sigma_{i}\left(U \cup N_{i}\right)$. Here we use the special properties of $U$. For instance we could construct $\sigma_{i}$ by letting $\sigma_{i}(x, 0)=\left(x, 0,0, \pi_{i}(x)\right)$ for all $x \in U^{I} \subset V \times R^{n}$ and letting $\left.\sigma_{i}\right|_{N_{i}}=h_{i}$. We may extend this $\sigma_{i}$ to a neighborhood of $U \cup N_{i}$. Using the fact that there is an isotopy of $V \times R^{n} \times R \times R^{a} \times R^{m+1}$ whose time one map restricted to $N_{i} \times 0 \times 0$ is $h_{i}$, we may extend $\sigma_{i}$ to all of $N$. Now by Lemma 2.9' we may isotop $\sigma_{i}(N)$ to a nonsingular algebraic set fixing $\sigma_{i}\left(U \cup N_{i}\right)=\sigma_{i}(U) \cup Y_{i}^{\prime}$. Hence we may assume in fact that $\sigma_{i}(N)$ is a nonsingular algebraic set $Y_{i}$.

Now by applying Lemma 2.9 to the diagonal map $N \rightarrow Y_{1} \times Y_{2} \times \cdots \times Y_{k}$ (recall $N \approx Y_{i}$ ) there is an integer $b$, a small isotopy $h_{t}^{\prime}$ of $V \times R^{n} \times R \times R^{b}$ fixing $U \times 0$, an algebraic set $Z \subset V \times R^{n} \times R \times R^{b}$ and an entire rational function $u=\left(u_{1}, \ldots, u_{k}\right): Z \rightarrow Y_{1} \times Y_{2} \times \cdots \times Y_{k}$ so that $h_{1}^{\prime}(N \times 0)=Z^{\prime}$ is a nonsingular component of $Z, u_{i}(x, 0)=\sigma_{i}(x)$ for all $x \in U$, and the $u_{i} h_{1}^{\prime}$ are closed approximations to the $\sigma_{i}, i=1, \ldots, k$. By Lemma 2.6 there is a small isotopy in $Z^{\prime}$ taking $U \times 0 \cup h_{1}^{\prime}(M \times 0 \times 0 \times 0)$ to a nonsingular projectively closed algebraic subset $X^{\prime}$ of $Z$ so that $U \times 0 \subset X^{\prime}$. But then by Lemma $2.3 X=X^{\prime}-U \times$ 0 is a projectively closed nonsingular algebraic set. 
The fact that the $u_{i} h_{1}^{\prime}$ are close approximations to $\sigma_{i}$ means that there is a small isotopy from each $u_{i}^{-1}\left(Y_{i}^{\prime}\right) \cap Z^{\prime}$ to $h_{1}^{\prime}\left(N_{i} \times 0\right)=h_{1}^{\prime} \sigma_{i}^{-1}\left(Y_{i}^{\prime}\right)$. Also $u_{i}^{-1}\left(Y_{i}^{\prime}\right) \cap Z^{\prime}$ consists of nonsingular points of dimension $\operatorname{dim} N-c_{i}$ by Lemma 2.2. Hence by Lemma 2.1 there is a small ambient isotopy $h_{t}^{\prime \prime}$ of $Z^{\prime}$ so that $h_{1}^{\prime \prime} h_{1}^{\prime}\left(N_{i} \times 0\right)=$ $u_{i}^{-1}\left(Y_{i}^{\prime}\right) \cap Z^{\prime}$ and $h_{1}^{\prime \prime} h_{1}^{\prime}(M \times 0 \times 0 \times 0)=X$. Let $X_{i}=u_{i}^{-1}\left(Y_{i}^{\prime}\right) \cap X$. Notice that $X_{i}$ are nonsingular since they are the transverse intersections of nonsingular components of algebraic sets. Also $X_{i}=h_{1}^{\prime \prime} h_{1}^{\prime}\left(M_{i} \times 0 \times 0 \times 0\right)$. Therefore we have proven the theorem.

\section{REFERENCES}

[A] S. Akbulut, Algebraic equations for a class of P.L. spaces, Math. Ann. 231 (1977), 19-31.

$\left[\mathrm{AK}_{1}\right]$ S. Akbulut and H. King, Real algebraic variety structures of P.L. manifolds, Bull. Amer. Math. Soc. 83 (1977), 281-282.

$\left[\mathrm{AK}_{2}\right] \_$, Topology of real algebraic sets with isolated singularities, Ann. of Math. 113 (1981).

[AK $\left.{ }_{3}\right] \ldots$, All knots are algebraic, Comment. Math. Helv. 56 (1981).

[CF] P. Conner and E. Floyd, Differentiable periodic maps, Ergebnisse der Mathematik und ihrer Grenzgebiete, Band 33, Springer-Verlag, Berlin, 1964.

[H] H. Hironaka, Resolution of singularities of an algebraic variety over a field of characteristic zero, Ann. of Math. 79 (1964), 109-326.

[L] S. Lojasiewicz, Sur le probleme de la division, Rozprawy Math. 22 (1961), 1-57.

[M] J. Milnor, On the Steifel-Whitney numbers of complex manifolds and of spin manifolds, Topology 3 (1965), 223-230.

[N] J. Nash, Real algebraic manifolds, Ann. of Math. 56 (1952), 405-421.

[T] A. Tognoli, Su una congettura di Nash, Ann. Scuola Norm. Sup. Pisa Sci. Fis. Mat. 27 (1973), $167-185$.

School of Mathematics, The Institute for Advanced Study, Princeton, New Jersey 08540

Department of Mathematics, University of Maryland, College Park, Maryland 20742 